\title{
Spatio-temporal analysis of color Doppler information using independent component analysis
}

\author{
Yasser M. Kadah ${ }^{*}$ \\ Biomedical Engineering Department, Cairo University, Giza, Egypt
}

\begin{abstract}
Observing the correlation between the information in neighboring resolution cells, we propose a methodology to allow better analysis of Doppler ultrasound signals. A blind source separation problem is formulated to discern the different signal components for correct interpretation of the data using independent component analysis (ICA). The Doppler signal is modeled as the summation of the true velocity signal, baseline fluctuation, and random noise. The baseline fluctuation component can be considered as a deterministic yet unknown signal. A simple adaptive denoising technique is applied to reduce the effective dimension of the noise subspace. Then, given a region of interest, the temporal signals corresponding to all pixels within this region undergo the ICA iteration to compute a set of independent signals that most represent the actual components present within the data. Subsequently, a comparison of the temporal variations of these signals allows the user identify the components of the signals that correspond to baseline variation or random noise manually or using semi-automated techniques. The new technique shows large potential to alleviate some of the limitations in this demanding imaging mode as well as to make the interpretation of the results more robust.
\end{abstract}

Keywords: Color Doppler, Denoising, independent component analysis

\section{INTRODUCTION}

The use of ultrasound imaging has become wide spread in current clinical diagnostic procedures. It enables the structures of the human body to be imaged noninvasively without exposing the patient to any form of health hazards. Among the most advanced aspects of this technology is its use to obtain maps representing blood flow characteristics based on the Doppler principle ${ }^{1}$. Such maps represent flow velocities or power in different colors overlaid on the conventional B-mode anatomical images, and that is why it is called color flow mapping (CFM). Such maps can be computed as some measure of blood flow velocity or the power computed from integrating over all Doppler shifts. This is a particularly demanding task that requires fast acquisition of large sets of data as well as a substantial amount of computational effort to produce real-time display of such information at a reasonable accuracy.

Even though the theory and methodologies used in the area of CFM have been the center of focus of Doppler research groups in the past two decades, it is not straightforward to implement such techniques on clinical systems. Given an ultrasound system with the necessary acquisition and computational requirements for CFM, the computed CFM images may not yield useful information results in many cases as a result of signal contamination with both random and colored noise. The solution most implementations resort to is to fine-tune the filtration processes until satisfying results are obtained. This requires a lot of effort with the disappointing result that many such implementations are considered not robust by users. Therefore, a strategy that allows a more robust analysis of such information would be rather useful in making this technology more accessible.

We observe that the CFM data set used to generate a single instance of the CFM output bears similarity in many aspects to the data used in other medical imaging areas such as event-related studies performed using functional magnetic resonance imaging ${ }^{3-6}$. In such studies, the outcome of the experiment is in the form of a collection of temporal signals corresponding to different spatial positions that characterized by a very low signal-to-noise ratio (SNR). Given that a number of techniques have been proposed to handle such data adaptively while preserving the true signal part of the measured signal ${ }^{3-6}$. Among those approaches is the use of principal and independent component analyses in resolving the different sources that contribute to the measured signal ${ }^{4}$. Given the similarities with the CFM problem, it is of interest to investigate the use of such source separation techniques in this problem. In particular, we would like to be

\footnotetext{
*E-mail: ymk@ieee.org
} 
able to compute the different sources that contribute to the CFM data and use only those which contain the true signal in calculating the CFM images. Once this is possible, it is possible to compute more accurate maps and suppress artifacts resulting from other unwanted sources.

In this work, we study the problem of analyzing the CFM data using blind source separation techniques such as ICA. A model for the CFM data is proposed as the sum of a deterministic part and a random noise part. We propose the use of a simple adaptive denoising procedure as a preprocessing step to satisfy the fundamental requirements of ICA. Then, ICA is used to generate a set of signals corresponding to the underlying sources in the signal. We demonstrate the ability of such analysis to separate the true signal from other unwanted deterministic signal components such as wall motion. Once this analysis is finalized, individual temporal signals can be projected onto the space corresponding to the true signal and only this information is used to generate the CFM image. This approach has the potential of making the CFM procedure more robust for clinical settings as well as to provide an implicit means for averaging the temporal data using its spatial variations.

\section{SIGNAL MODEL}

In color flow mapping, the image is constructed within the region of interest using estimates of some blood velocity measure in each pixel. Hence, to maintain the accuracy of the map, robust measures have been proposed in the literature to lower the sensitivity of estimates in the presence of noise. We propose a different strategy in this work that uses two steps; a modeling step and an analysis step. The advantage of this strategy is a more robust performance in the presence of different types and levels of random and colored noise.

The model of the data collected to reconstruct a single instance of the color flow map consists of a three dimensional complex array consisting of the quadrature samples collected from the 2-D region of interest along a specified time window. We consider now the time course signals of individual pixel. In conventional CFM techniques, each of these time courses is used to compute a single value to describe the velocity at this specific spatial position and time ${ }^{2}$. Here, we try to decompose this signal into its underlying components before we attempt to compute such estimate.

Each time course is assumed to contain contributions from four independent components: the true Doppler signal, the signal resulting from wall motion, clutter and baseline variation, and random noise ${ }^{1,2}$. The first three components are considered as deterministic yet unknown signals. The wall motion signal can be suppressed by using an analog wall motion filter before sampling of the signal. However, a residual contribution from this component usually remains with the filtered signal. In the more advanced Doppler system, wall motion filtering can be performed on the digital side using a high-resolution analog-to-digital converter to accommodate the required large dynamic range. This component is modeled as a large magnitude low frequency signal superimposed on the original signal. The third component is present as a result of the presence of stationary structures within each voxel. On the other hand, the fourth component is assumed to consist primarily of thermal noise that belongs to a zero-mean white Gaussian noise on both quadrature parts of the signal. The velocity estimate should be computed using only the first component.

Given that the contribution of each of the four components to the measured signal vary between different pixel locations, it is not generally possible to using simple averaging techniques to improve the accuracy of the map. The approach we propose here to improve the accuracy is to take advantage of the availability of time course signals from different spatial locations and the common origin and form of such signals as the wall motion signal and clutter signal among them. In particular, instead of filtering the individual signals using a rigid model for the deterministic and random nuisance sources, a nonparametric approach is used where the underlying sources of signal in the whole data set are estimated then used to remove nuisance from individual time courses. This approach relies on blind source separation methods such as principal component analysis or independent component analysis to perform the source localization from the data set at hand based on statistical criteria and without intervention from the user.

\section{INDEPENDENT COMPONENT ANALYSIS}

As mentioned above, the independent component analysis (ICA) belongs to a class of techniques that are commonly termed blind source separation techniques. A useful approach to understanding ICA starts with the principal component analysis. Principal component analysis (PCA) is analogous to Fourier analysis in that the data is described in terms of an orthogonal set of vectors. But rather than using complex exponentials, the orthogonal set in PCA is chosen so that it 
describes the samples most efficiently using the smallest number of terms. Hence, PCA is an efficient technique for dimensionality reduction in multivariate statistical analysis.

PCA derives the direction of a set of orthogonal vectors that point into the direction of the highest variance of the data set. The principal components are calculated as the eigenvectors of the covariance matrix of the data set. The eigenvalues denote the variance that corresponding principal components (i.e., eigenvectors) account for. The methodology consists of two distinct stages, that of defining the principal components of the study population, and that of calculating the coefficients of the principal components for each sample. PCA is defined as the eigen-decomposition of the covariance matrix. The output of the PCA transform is uncorrelated vectors (thatis, a second order statistic is used to compute the outcome of this analysis). That is, the covariance matrix of the output is a diagonal matrix. In general, principal components analysis results in a dimensionality reduction either naturally or by truncating eigenvectors corresponding to very small eigenvalues. The efficiency of this approximation can be estimated by the ratio between the sum of included eigenvalues to the sum of all eigenvalues.

Independent component analysis (ICA) is an extension of principal component analysis (PCA) whereby higher order statistics rather than second order moments are used to determine the basis vectors that are statistically as independent as possible ${ }^{7}$. This is the reason for selecting ICA rather than PCA for this problem as a result of its generality. A fast fixed-point algorithm (FastICA) is used for implementing ICA ${ }^{8}$. In FastICA, the data vectors are first whitened (i.e., a transformation is applied such that the variance values in all directions are made the same). The whitening step is performed using PCA. Besides whitening the data, PCA is used to reduce dimensionality (e.g., from n to q). Several approaches can then be taken to utilize the higher-order information. A principled approach is given by finding linear combinations of maximum non-Gaussianity, as motivated by the central limit theorem. Sums of independent random variables tend to be closer to Gaussian than the original ones. Therefore if we take a linear combination of the whitened variables, this will be maximally non-Gaussian if it equals one of the independent components. Non-Gaussianity is measured by many ways. Many researchers suggested solutions to the ICA problem using the fourth-order cumulant or kurtosis $^{7}$. Kurtosis is zero for Gaussian random vectors; for densities peaked at zero, it is positive, and negative for flatter densities. The kurtosis criterion is maximized by applying the iterative FastICA algorithm. To estimate m independent components, the algorithm iterates $\mathrm{m}$ times. To ensure that a different independent component is estimated each time, an orthogonalizing projection is added to the iteration.

It should be noted here that the use of complex signals in PCA or ICA computations is not possible. Therefore, the above methodology is applied to the real and imaginary parts independently. This complicates the interpretation of the results a little bit because of the need to fine one-to-one correspondences between the results of the two parts of the signal. Nevertheless, finding such information is rather straightforward and can be done using a simple comparison of the maximum cross-correlation between signals. Moreover, among the limitations of PCA and ICA is the constraint that the number of underlying independent sources must be less than the number of time course signals. This constraint is not satisfied in practical CFM situations as a result of the presence of a number of independent random signals that is equal to the number of signals with a few more to add corresponding to the deterministic parts of the signal. This severely limits the usefulness of the results of such techniques as well as the stability of the ICA iteration in particular. Therefore, we propose to utilize an adaptive denoising step before PCA and ICA to effectively reduce the dimension of the random noise subspace.

\section{SIGNAL DENOISING}

Given the type of components within the CFM temporal signal as described above, we will consider a model that is composed of the sum of one deterministic component $d(t)$ incorporating both the true Doppler, wall motion and clutter signals and an uncorrelated stochastic random noise component $n(t)$. That is,

$$
s(t)=d(t)+n(t)
$$

Since these two component are assumed independent, the corresponding power spectrums are related by,

$$
P_{s s}(\omega)=P_{d d}(\omega)+P_{n n}(\omega)
$$


where cross-terms vanish because the two components are assumed uncorrelated. Hence, an estimate of the power spectrum of the deterministic component takes the form ${ }^{9}$,

$$
P_{d d}(\omega)=P_{s s}(\omega)-P_{n n}(\omega)
$$

That is, the signal power spectrum is obtained by spectrum subtraction of the noisy signal and noise power spectra. In order to compute the deterministic signal component from its power spectrum, the magnitude of the Fourier transform can be obtained as the square root of the power spectrum. The problem now becomes that of reconstructing the signal using magnitude only information about its Fourier transform. Several techniques can be used to do that. The one used for this work relies on an estimate obtained from the phase of the Fourier transform of the original signal $S(\omega)$. Hence, the Fourier transform of the processed signal $S_{d}(\omega)$ can be expressed as,

$$
S_{d}(\omega)=\sqrt{P_{d d}(\omega)} \cdot \mathrm{e}^{j \operatorname{Phase}(S(\omega))}
$$

The enhanced deterministic signal $s_{d}(t)$ is then computed as the real part of the inverse Fourier transformation of this expression.

\section{METHODOLOGY}

\subsection{Adaptive parameter estimation of the noise model}

According to the above derivation, we need to compute the variance of the data in order to obtain the noise power spectrum model using the empirical formula. The simplest way to do that is to estimate the variance of background areas within the available data set. Background areas correspond to stationary tissues within the region of interest or can be estimated separately as the signal when the receiver multiplexer is turned OFF.

Since the proposed technique is applied to a single time course at a time, the periodogram estimate of signal power spectrum is expected to have a rather large variance ${ }^{10}$. As a result, the subtraction of power spectra in Eq. (3) may contain negative values in practical implementations. This causes a problem in trying to compute the square root to recover the processed signal. The approach used in our implementation to overcome this problem relies on replacing all negative values in the subtraction results by zero. This approach is justified because all values lower than the estimated power spectrum are more likely to be noise components within the variance limits of the periodogram estimate.

\subsection{Statistical noise removal}

Given the nature of the original signal, we observe that the variance in the power spectrum estimate may only result from the random component. Since the expected value of the noise variation is known from the derived model and given the statistical characteristics of the periodogram estimate, we can express the noise at each of the power spectrum frequency bins as a Gaussian random variable with mean and variance both equal to the noise model ${ }^{10}$. As a result, the subtraction in Eq. (3) would effectively remove only a part of the noise power spectrum. In other words, the upper half of the Gaussian distribution would still remain in the processed signal.

To solve this problem, a slight modification to the technique is added to allow direct control over the extent of noise removed. The modified equation takes the form,

$$
P_{d d}(\omega)=P_{s s}(\omega)-\alpha \cdot P_{n n}(\omega)
$$

Here, the factor $\alpha$ is added to control the confidence of noise removal. This problem can be expressed in the form of a statistical z-test where $\alpha$ controls the p-value of the test. That is, the larger the value of $\alpha$, the less the probability that the output power spectrum contains a noise component. On the other hand, increasing this value would increase the likelihood that some parts of the signal may also be removed. Therefore, the selection of the value of $\alpha$ is useful to finetune the results of the new technique. Several optimization criteria can be used to select the value of this parameter. An example of these is the use of entropy based objective function optimized over the autocorrelation function of the difference between the original and processed signals for different $\alpha$ values. This favors the values of $\alpha$ that give an autocorrelation function with narrow extent around zero and of minimal side peaks. This tends to preserve the 
components of the true signal, which give rise to periodic peaks in the autocorrelation function. Another approach is to optimize the kurtosis of the difference as close as possible to zero to make sure that the removed signal is only the Gaussian random noise component. In this work, we used a fixed value of this parameter that is equal to 1 to make it easier to compare the results and assess the improvement after using this technique as a preprocessing stage and the optimization of this parameter will be left for further investigation.

\subsection{Analysis of Results Using ICA}

To show the improvement in using ICA on the processed signal, the new technique is applied to process all pixel time courses in the acquired data set independently and then the processed data set is used for subsequent ICA. The PCA and ICA techniques were performed on the time courses of local regions using a Matlab (Math Works, Inc.) program based on FASTICA ${ }^{8}$. The goal of this analysis is to assess the performance of the new technique in enhancing the results of PCA and ICA and stabilizing the convergence characteristics of the ICA. Moreover, the difference signals between the original and filtered data sets were also analyzed using these techniques. This helps verify the absence of signal components within this discarded part of the original signal.

\section{RESULTS AND DISCUSSION}

The proposed method was implemented to compute the ICA of the proposed model consisting of a Doppler shift signals (assumed to include a range between $300 \mathrm{~Hz}$ and $3 \mathrm{kHz}$ ), a wall motion signal (assumed around 100Hz), a baseline shift signal (a signal around $1 \mathrm{~Hz}$ ), and a random white Gaussian noise component. The real and imaginary parts of the signal were preprocessing using the denoising method and then analyzed using ICA. The original signals are shown in Figure 1. The results from analyzing the original signal (before denoising) using PCA are shown in Figure 2 while those of the ICA are shown in Figure 3. The results after the denoising process are shown in Figures 4 and 5. As can be seen, there is a marked improvement on the accuracy of the analysis results in the denoised signals.

It was observed from our experiments that the results of ICA correspond more closely to the actual signal components unlike PCA, which varied substantially and resulted in combinations of signals from different sources. Moreover, we notice that the analysis results from the ICA technique were rather robust when the individual component contributions are varied to increase the unwanted components. This indicates the potential of such method for this application.

The computational complexity of the proposed technique is obviously high compared to the requirements of real-time display of CFM information. At this point, we propose to use the technique as an off-line tool to analyze the CFM data and to possibly generate a CINE replay of the acquired data at a higher accuracy. This issue has to be addressed in more depth and detail in future work.

\section{CONCLUSIONS}

A new approach for color flow mapping data analysis was proposed. We observe that the deterministic parts of the time course signals in this application originate from a small number of sources. As a result, a denoising strategy is used to suppress the random noise subspace before applying source separation techniques to estimate such sources. Once these sources are localized, the ones that correspond to unwanted noise are marked and removed from all time courses before the color flow mapping image is computed. This results in a more robust performance for the system.

\section{REFERENCES}

1. D. H. Evans and W. N. McDicken, Doppler Ultrasound Physics, Instrumentation and Signal Processing, $2^{\text {nd }}$ ed., John Wiley \& Sons, New York, 2000.

2. J. A. Jensen, Estimation of Blood Velocities Using Ultrasound A Signal Processing Approach, Cambridge University Press, Cambridge, 1996.

3. Yasser M. Kadah, "Adaptive signal-preserving denoising of ER-fMRI data using spectrum subtraction," Proc. ISMRM/ESMRMB Joint Annual Meeting, Glasgow, Scotland, April 2001.

4. S. Makeig, T. Jung, D. Ghahremani, and T.J. Sejnowski, Independent component analysis of simulated ERP data, Institute for Neural Computation Technical Report No. INC-9606. 
5. S. Makeig, M. Westerfield, T. Jung, J. Covington, J. Townsend, T.J. Sejnowski, and E. Courchesne, "Functionally independent components of the late positive event-related potential during spatial attention," J. Neuroscience 19, no. 7, pp. 2665-2680, 1999.

6. M.J. Mckeown, T. Jung, S. Makeig, G. Brown, S.S. Kindermann, T. Lee, and T.J. Sejnowski, "Spatially independent activity patterns in functional MRI data during the Stroop color-naming task," Proc. Natl. Acad. Sci., USA 95, pp. 803-810, 1998.

7. A. Hyvarinen, J. Karhunen, and E. Oja, Independent Component Analysis, John Wiley \& Sons, New York, 2001.

8. http://www.cis.hut.fi/projects/ica/fastica.

9. S.V. Vaseghi, Advanced Signal Processing and Digital Noise Reduction, John Wiley \& Sons, New York, 1996.

10. C.S. Burrus, J.H. McClellan, A.V. Oppenheim, T.W. Parks, R.W. Schafer, H.W. Schuessler, Computer-Based Excercises for Signal Processing Using Matlab, Prentice Hall, New Jersey, 1994.

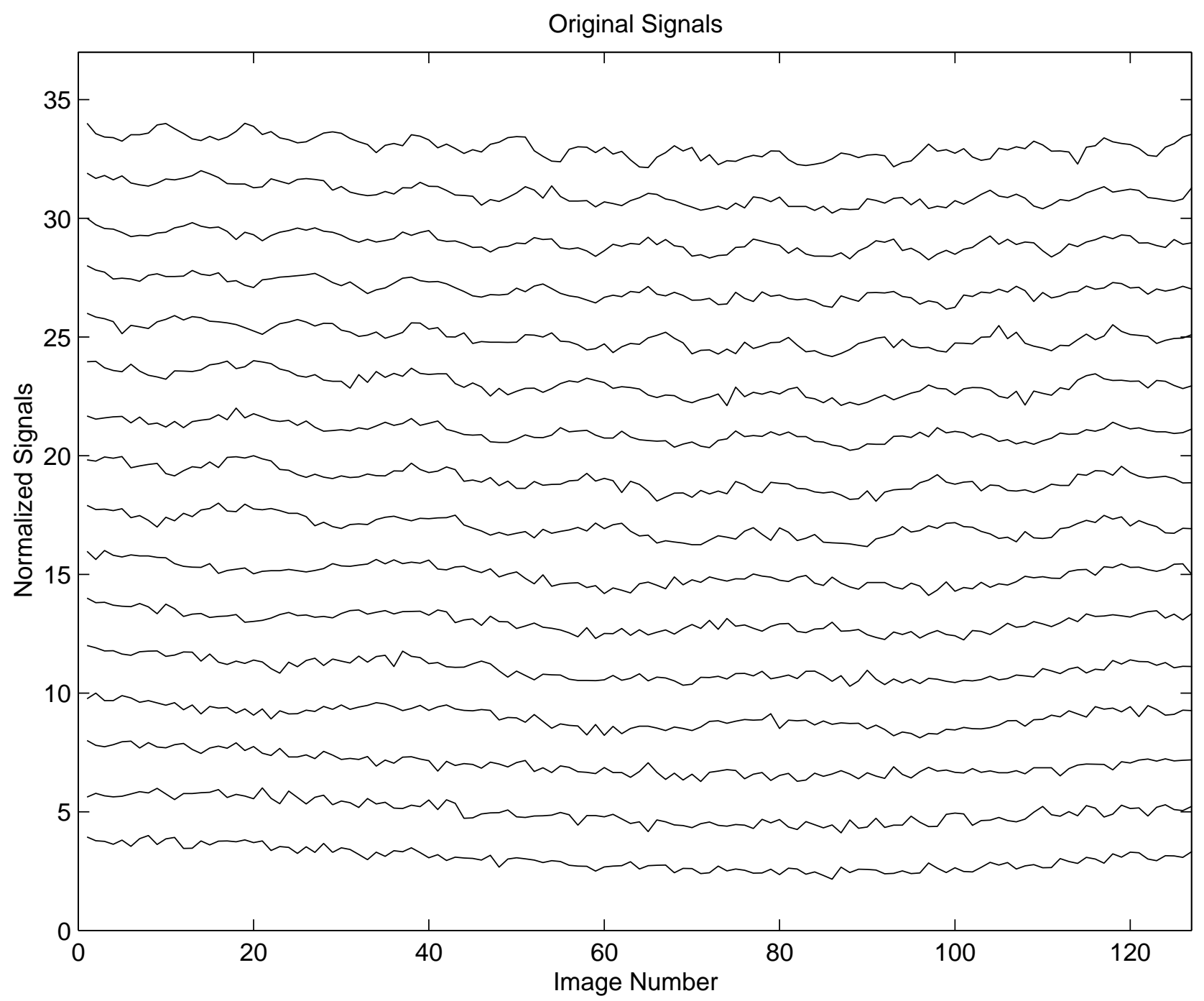

Figure 1. A plot of the original CFM time course data. 


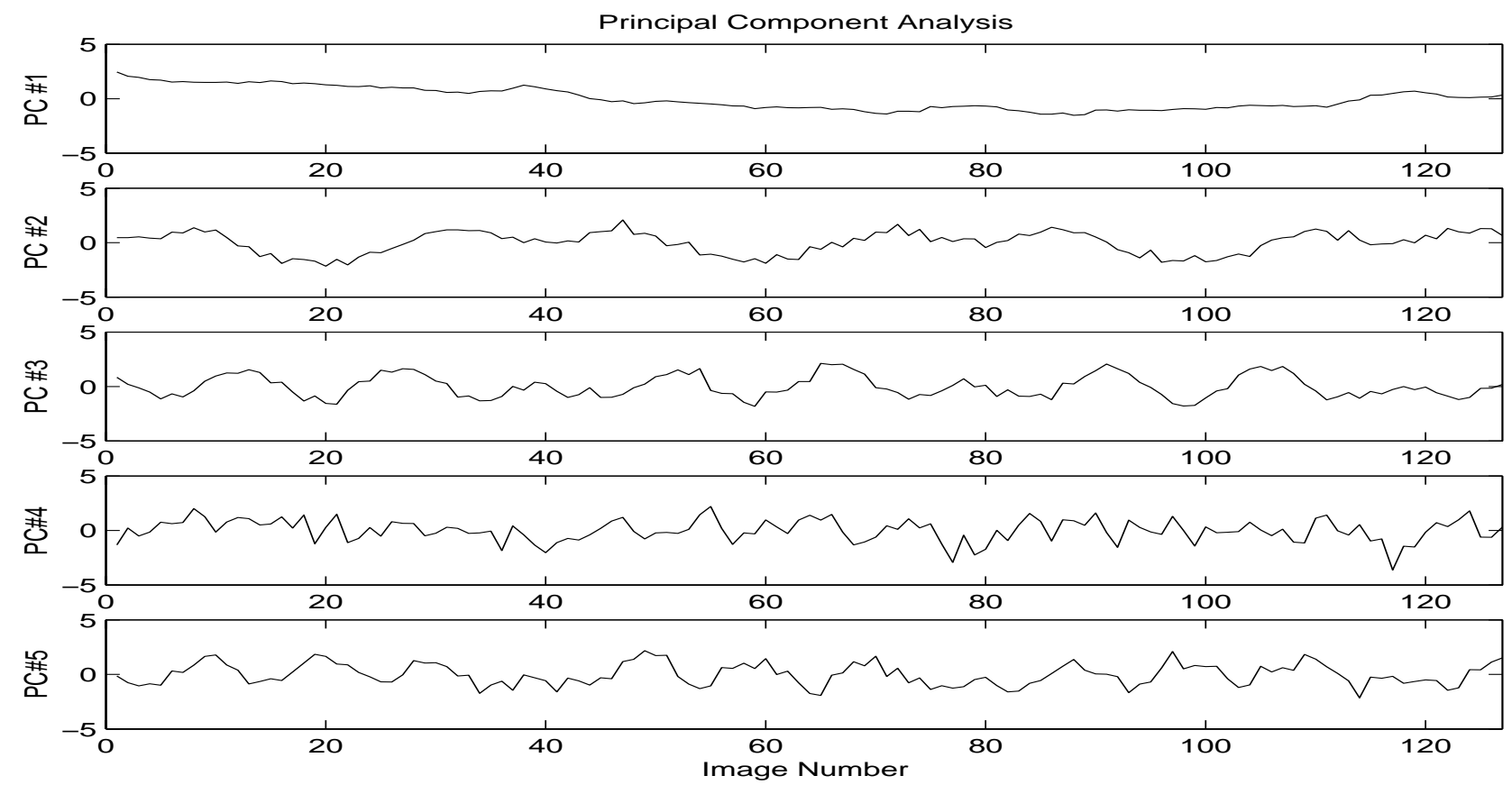

Figure 2. PCA for original data.
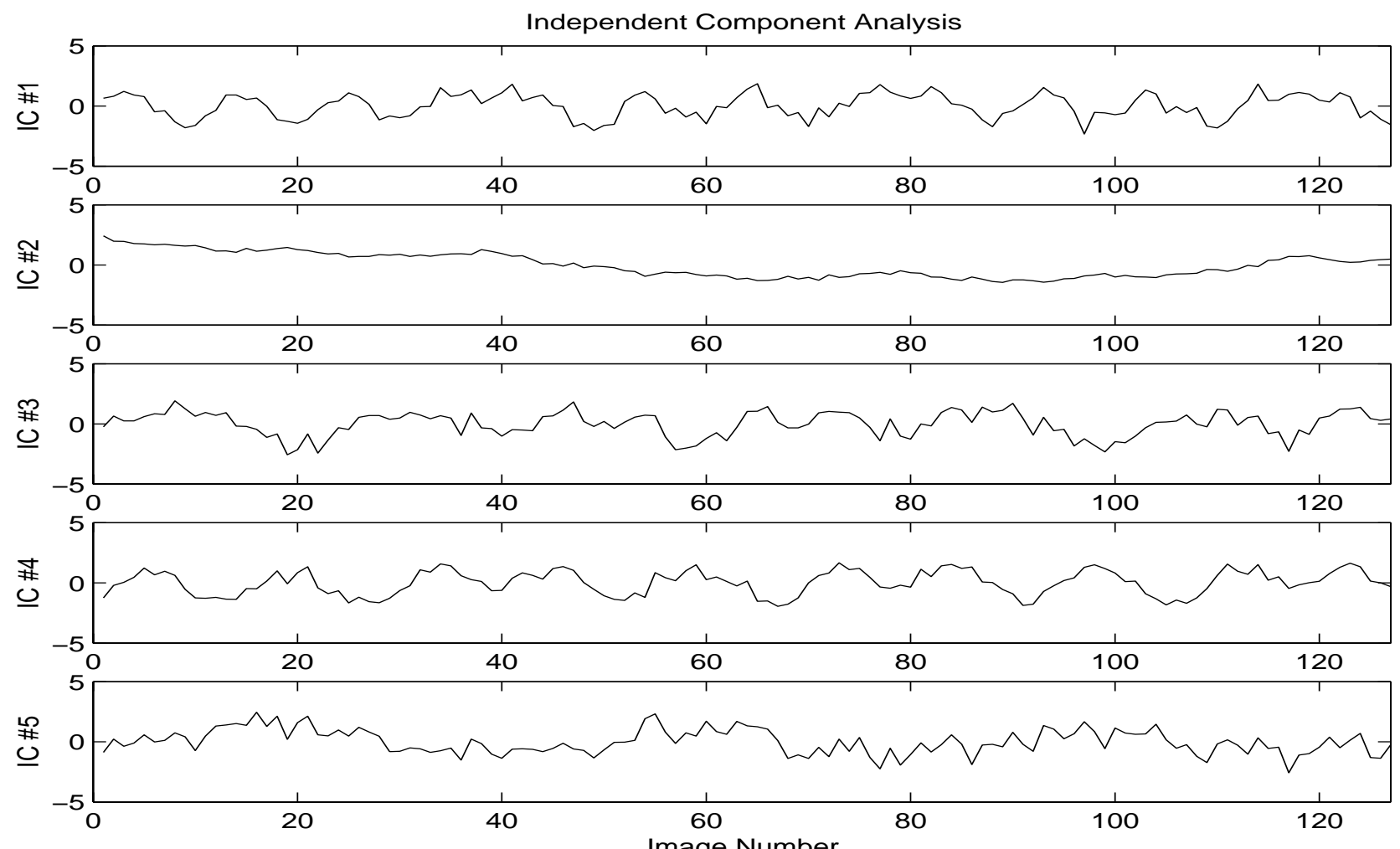

Figure 3. ICA for original data. 

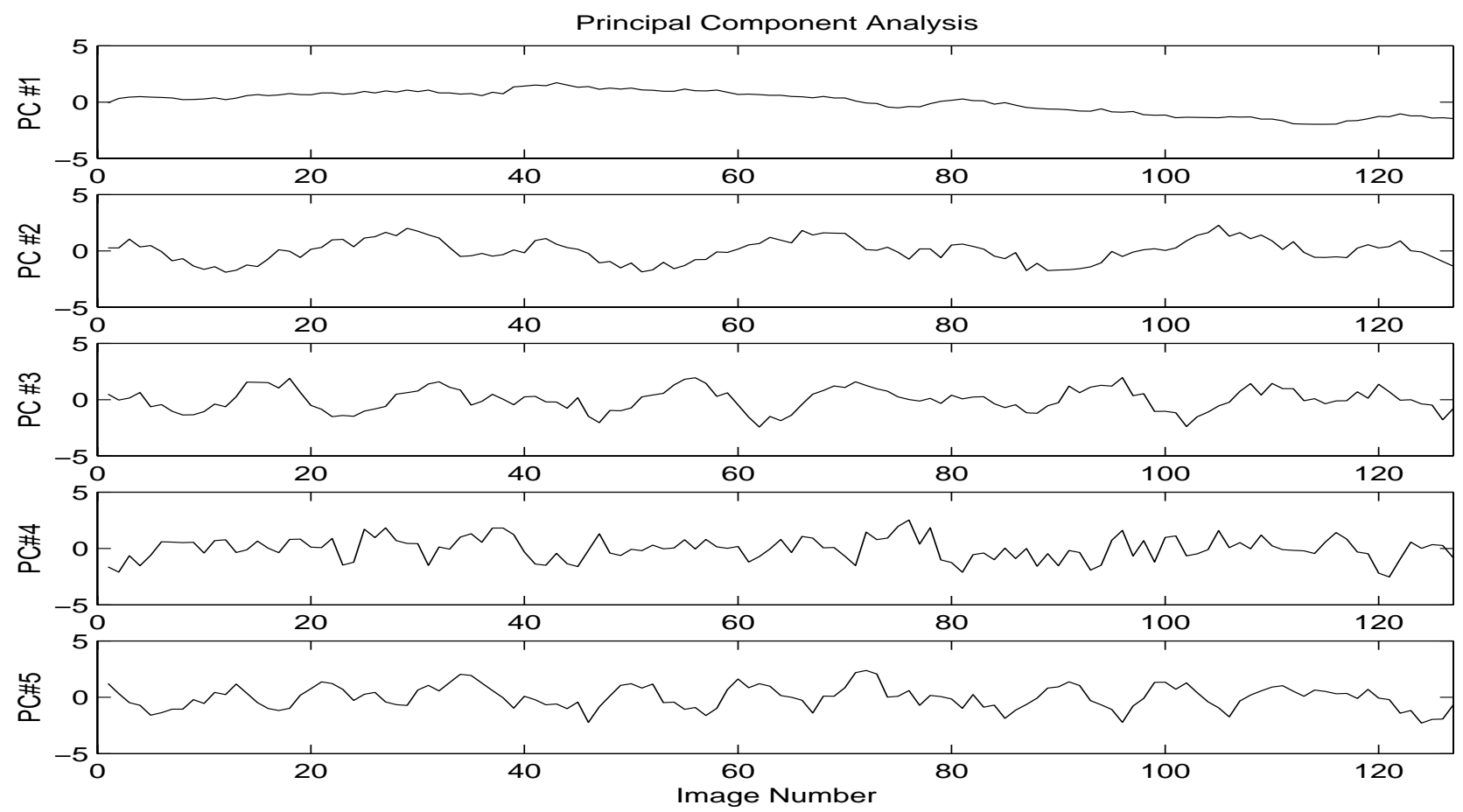

Figure 4. PCA for denoised data.
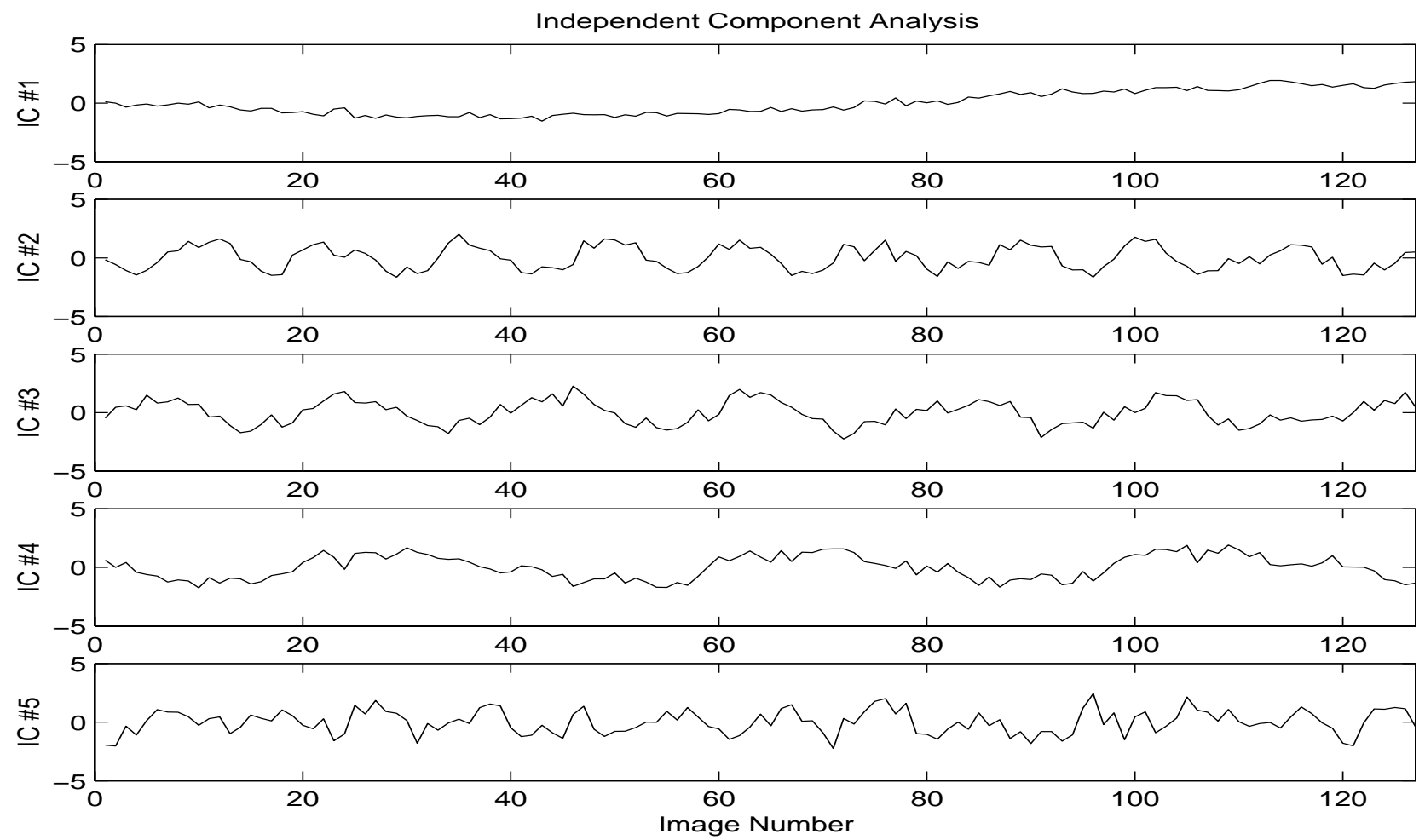

Figure 5. ICA for denoised data. 